\title{
CLINICO-EPIDEMIOLOGICAL PROFILE OF BRAIN ABSCESS- A SINGLE CENTER STUDY
}

\begin{tabular}{ll} 
Jagminder Singh & Assistant Professor, Department of Neurosurgery, DMC\&H, Ludhiana \\
\hline Monique Garg & $\begin{array}{l}\text { Associate Consultant, Department of Neurosurgery, MAX Hospital, } \\
\text { Shalimar Bagh, Delhi }\end{array}$ \\
\hline Shivender Sobti* & $\begin{array}{l}\text { Associate Professor, Department of Neurosurgery, DMC\&H, Ludhiana } \\
{ }^{*} \text { Corresponding Author }\end{array}$ \\
\hline Ajay Choudhary & $\begin{array}{l}\text { Professor \& HOD, Department of Neurosurgery, ABVIMS, DR.RML Hospital, } \\
\text { New Delhi }\end{array}$ \\
\hline Rupinder Kaur & Assistant Professor, Department of Medicine, DMC\&H, Ludhiana \\
\hline
\end{tabular}

ABSTRACT INTRODUCTION: The overall incidence of bacterial brain abscess has remained relatively constant despite improved treatment of underlying systemic infections and development of more effective antibiotics. The systemic antibiotics are generally given for 6-8 weeks. The Choice of surgery varies from stereotactic aspiration /open surgical method including twist drill aspiration, burr hole aspiration with or without drainage, small craniectomy with aspiration and drainage to craniotomy and excision of abscess. The purpose of this article is to share authors' experience regarding clinico epidemiological profile of brain abscess.

MATERIAL AND METHODS: Total 30 patients with brain abscess who presented to the Department of Neurosurgery, PGIMER Dr. RML Hospital Delhi, from November 2016 to April 2018 analyzed for clinical, epidemiological, microbiological profile of brain abscess patients along with modes of treatment and their outcome.

RESULTS: Total 30 patients of Brain Abscess were evaluated with males outnumbering females. Predominated symptoms were fever $(96.7 \%)$, headache $(83.3 \%)$ and nausea and vomiting $(40 \%)$. The chronic suppurative otitis media (CSOM) was predominately underlying factor in 18 patients i.e. (60\%) and commonest organism isolated was streptococcus pneumonia (66.6\%) followed by streptococcus Milleri (33.33\%). The length of hospital stay was longer in aspiration group $\{21.2 \mathrm{days}$ (SD \pm $4.1)\}$ compared to excision group $\{13$ days (SD \pm 1.1$)\}$. Total $20(66.7 \%)$ patients survived, $2(6.7 \%)$ patients died and $8(26.7 \%)$ were lost to follow up.

CONCLUSION: Majority of time there is an underlying factor for brain abscess. The treatment of brain abscess involves both medical and surgical modalities. Third generation cephalosporins and metronidazole are the most commonly used antimicrobial agents in the treatment of brain abscesses. The length of hospital stay in excision group was less as compared to medical group and aspiration group.

\section{KEYWORDS : Brain abscess, CSOM, Medical management, Aspiration, Excision.}

\section{INTRODUCTION}

A brain abscess is an intraparenchymal collection of pus. The incidence of brain abscess is approximately $8 \%$ of intracranial masses in developing countries and $1-2 \%$ in the western countries ${ }^{(1,2)}$. The changes in epidemiology and clinical spectrum of the brain abscess, the predisposing factors and the prevalence of implicated bacterial pathogens contribute to mortality in different rates. The overall incidence of bacterial brain abscess has remained relatively constant despite improved treatment of underlying systemic infections and development of more effective antibiotics ${ }^{(3)}$. Cases are spread out throughout the population mostly seen in first two decades of life, peak incidence being 4-7 years, with evolution of bacteriological profile overtime in past thirty years with abscesses due to staphylococcus decreasing, streptococcus remaining same and abscesses due to gram negative bacteria increasing. There continues to be a strong representation of anaerobes (30 to $50 \%$ ) in patients with brain abscess ${ }^{(1)}$. The brain abscess is almost always secondary to a focus of suppuration elsewhere in the body and may develop either by spread from a contiguous focus of infection, after neurosurgery or head trauma or by hematogenous spread from a distant focus ${ }^{(4,5)}$. The management of brain abscesses aims to reduce the space-occupying activity, reduce the intracranial pressure, and eradicate the pathogenic microorganism. The anatomical location, number and size of abscesses, stage of abscess formation, age and neurological status of the patient can influence the strategy for managing brain abscess ${ }^{(6)}$

This study reviewed the key aspect of the clinical presentation of brain abscess as well as recent advances in the diagnosis and treatment of the condition.

\section{MATERIAL AND METHODS}

The study included 30 patients presenting to the Department of Neurosurgery, PGIMER Dr. RML Hospital Delhi, with a likely diagnosis of brain abscess from November 2016 to April 2018. A detailed history, physical examination and blood investigations of enrolled patients were done. Preoperative plain and contrast Computer tomography $(\mathrm{CT})$ was done in all patients. Magnetic resonance imaging (MRI) performed whenever diagnosis was in doubt. Underlying source of infection and predisposing factors were identified and analyzed. The patients were categorized on the bases of treatment given i.e. medical treatment alone or in combination with one or other surgical mode of management. Small acute and subacute stage brain abscesses (defined as $<2.5 \mathrm{~cm}$ in mean diameter) without consciousness disturbance were treated using antibiotics alone. Indications for surgery ( Burr hole with aspiration / craniotomy with excision) were neuroimaging demonstration of intracranial large brain abscess formation in capsule stage, presence of significant mass effect, high intracranial pressure, proximity to ventricles and possibility of ventricular rupture, posttraumatic, poor response to medical treatment, deterioration in neurological status, or enlargement of abscess volume during medical treatment. Direct excision of brain abscess was done if size $\geq 4 \mathrm{~cm}$. Antibiotic therapy was instituted after culture material had been obtained during surgery. Antibiotic therapy was continued for $\geq 6$ weeks in accordance with the therapeutic response and neuroimaging 
findings. CT or MR imaging was performed 24 hours after aspiration and as and when required. Repeated aspiration/excision was done if size of abscess increased or remains same after initial aspiration and antibiotics. Patients with poor response to repetitive aspirations and medical treatment underwent complete excision of abscesses through craniotomy or craniectomy. The Clinical and neuroradiological findings, treatment modalities, pus cultures and organisms grown, therapeutic outcomes were summarized.

\section{EXCLUSION CRITERIA:}

The patients with tubercular meningitis, tubercular abscess, subdural empyema, extradural abscess were excluded from the study.

\section{RESULTS}

Total 30 patients of Brain Abscess who were admitted and evaluated in the Department of Neurosurgery over a period of two years were included in the study. It was observed that majority of the patients in our study were males numbering $21(70 \%)$ whereas there were only 9 females (30\%). Majority of our patients age group was in the range of $10-20$ years forming $46.66 \%$ of the studied population and 2 nd most common age group was in range of $1-10$ years. The youngest patient being 1 month old and eldest patient was 58 years of age, with an average mean of years. More than one symptom seen in our patients, fever $(96.7 \%)$, headache $(83.3 \%)$ and nausea and vomiting $(40 \%)$ predominated among others. Only two of our patients i.e. (6.7\%) had a low score on Glasgow coma scale (GCS) <13 whereas majority of our patients, 28 i.e. (93.3\%) were alert at the time of admission with a GCS >13. It was observed that among the underlying factors CSOM predominated with 18 patients i.e. $(60 \%)$ having an underlying unsafe ear. Other underlying factors in order of frequency were post traumatic $3(10 \%)$, heart disease $2(6.7 \%)$, postcraniotomy l (3.3\%), sinusitis l (3.3\%). In 5 patients i.e. (16.7\%) no underlying factor could be identified and were thus labelled as cryptogenic. It was seen that among the patients who had CSOM as the underlying factor commonest organism isolated was streptococcus pneumonia 4(66.6\%) followed by streptococcus Milleri 2(33.33\%), followed by staphylococcus aureus and epidermidis $4(33.33 \%)$ and pseudomonas Aeruginosa and Enterobacter species 2(16.6\%) among the others organism isolated. In post-traumatic $2(66.6 \%)$ patients largest representing organism was from staphylococcus aureus and $1(33.3 \%)$ patient positive for Enterobacter. In post craniotomy patient staphylococcus aureus was the organism isolated and in patients having sinusitis as the cause for brain abscess staphylococcus epidermis was isolated. In cryptogenic group pseudomonas Aeruginosa and Enterobacter species 3 (75\%) combined together were the commonest organism isolated and in one patient streptococcus pneumonia was isolated. The temporal lobe was most common site for brain abscess ,12 patients (40\%) and frontal lobe abscess 6 patients (20\%) was second most common. The parietal, occipital, cerebellar and multiple sites abscess constitute 5 (16.7\%), 2 (6.7\%), 1 (3.3\%) and 4 (13.3\%) respectively. All the 30 patients received empirical medical therapy as the first form of management, however in 4 (13.3\%) medical therapy was continued as the sole form of treatment. In $26(86.7 \%)$ patients some form of surgery was added to the medical management already continuing. A total of $20(76.9 \%)$ patients received aspiration as a form of treatment, whereas $4(15.4 \%)$ patients underwent excision of their brain abscesses, $2(7.7 \%)$ patients received aspiration followed by excision of the brain abscess in combination. Among the 22 patients who underwent aspiration of their Brain abscess 14 i.e. (63.6\%) underwent aspiration once only, 6 patients i.e. (27.3\%) had to undergo aspiration twice whereas for 2 patients aspiration was repeated a third time. The 21 patients $(70 \%)$ had a positive culture while as 9 patients (30\%) did not grow any bacteria on culture. Most of cultures 18 (85.7\%) grew only single organism whereas 3 cultures grew 2 organisms. The commonest organism grown was Streptococcus Pneumonia 12 (57.1\%) followed by Streptococcus Milleri 6 (28.5\%), Staph Aureus 5 (23.8\%), Pseudomonas Aeruginosa 3 (14.3) \%) and others in smaller percentages. In 20 (66.7\%) patients specific I.V antibiotics were given where as in 10 (33.3\%) empirical antibiotics were continued. Majority of the patients 27 (90\%) received three antibiotics and in 3 patients (10\%) four antibiotics were used. I.V Ceftriaxone + Sulbactam was used in 25 (83.3\%), Amikacin in 20 (66.7\%) patients. Vancomycin 10 (33.3\%), ceftazidime 2 (6.7\%), I.V Metronidazole was used in all patients. It was observed that $5(16.6 \%)$ patients suffered wound infection as their commonest post-operative complication followed by Meningitis 4 (13.3\%), Sepsis 2 (6.7\%), Cerebritis 1 (3.3\%). Total $20(66.7 \%)$ patients were survived, $2(6.7 \%)$ patients died and $8(26.7 \%)$ were lost in follow up. The mean hospital stays for medically treated patients, aspiration group, aspiration plus excision and excision group was 37.2 days ( $\mathrm{SD} \pm 5.7), 21.2$ days ( $\mathrm{SD} \pm 4.1$ ), 17 days $(\mathrm{SD} \pm 1.4)$ and 13 days $(\mathrm{SD} \pm 1.1)$ respectively.

\section{DISCUSSION}

The overall incidence of pyogenic brain abscess in the developed nations is increasing with increased survival of patients with HIV, increasing number of transplant patients, and a proliferation of immunosuppressive regimens such as antineoplastic chemotherapy. In our study a preponderance of males over females (70:30) was found among our patients. These results were in concordance with results derived by Beller A J and colleagues ${ }^{(7)}$. with male over female preponderance of 60:29 and Cavusoglu $H$ and colleagues ${ }^{(6)}$ with male female preponderance of 36:15. It was observed that the commonest age group of our patients was $10-20$ years (46.66\%),youngest patient being 1 month old and oldest patient was 58 years of age, with an average mean of 31.2 years. Cavusoglu $\mathrm{H}$ and colleagues ${ }^{(6)}$ had patients ranging in age from 14 months to 58 years (median 29 years) at the time of diagnosis. Poffenbarger $\mathrm{G}$ J and colleagues ${ }^{(1)}$ concluded that the disease is overly represented among those in the first two decades of life. In our patients, fever (96.7\%), headache $(83.3 \%)$ and nausea and vomiting $(40 \%)$ were predominated symptoms. Our patients presented with multiple symptoms most of the time. This is in concordance with the study by Cavusoglu $\mathrm{H}$ and colleagues ${ }^{(6)}$ who derived almost the same results with headache (80\%), nausea and vomiting (44\%), fever $(74 \%)$ and neurological deficits (40\%). Our observations were consistent with those made by Poffenbarger GJ et al ${ }^{(1)}$

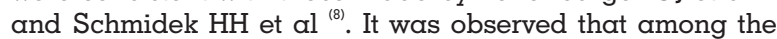
underlying factors CSOM predominated with 18 patients i.e. (60\%), post traumatic $3(10 \%)$, heart disease $2(6.7 \%)$, postcraniotomy l (3.3\%), sinusitis l (3.3\%). In 5 patients i.e. (16.7\%) no underlying factor could be identified and were thus labelled as cryptogenic. Our study is in consistence with the study of Beller AJ et al ${ }^{(7)}$ and Yang SY ${ }^{(4)}$ in view of source of infection either within or near the cranial cavity which observed $57 \%$ and $40-60 \%$ cases respectively. In our study the commonest organism grown was Streptococcus Pneumonia $12(57.1 \%)$ followed by Streptococcus Milleri 6 (28.5\%), Staph Aureus 5 (23.8\%), Pseudomonas Aeruginosa 3 (14.3) \%). Beller AJ et al ${ }^{(7)}$ Staphylococcus was the commonest organism cultured; it was found in $63 \%$ of the positive cultures. The second largest group were streptococci, found in almost $20 \%$ of the positive cultures. origin. Çavuşoglu et $\mathrm{al}^{(6)}$ observed that streptococcus $(36 \%)$, staphylococcus $(18 \%)$ were the commonest organisms cultured. In our study, majority of the brain abscesses were supratentorial in location (94\%) which is in complete harmony with the observation made by Poffenbarger GJ et al (1), in which $85 \%$ abscesses were supratentorial. Temporal (28.9\%) followed by temporoparietal region $(22.8 \%)$ were the commonest location of abscesses in 
our patients, however in series of patients observed by Çavuşoglu et al ${ }^{(6)}$ temporoparietal region $(24 \%)$ is the commonest location. In the study done by Beller $\mathrm{AJ}$ et al (7) temporal region was the commonest site involved with $30 \%$ of abscesses in that location. The most common surgical procedure used in our series was aspiration via a single burr hole in $22(73.3 \%)$ patients followed by excision in $6(20 \%)$ patients. In the aspiration group $6(27.7 \%)$ patients needed aspiration two times and in $2(9.1 \%)$ patients three times. Cavusoglu $\mathrm{H}$ et $a \mathrm{l}^{(6)}$ repeated aspiration more than once in 30 out 32 (93\%) patients who underwent aspiration in their series of 51 patients and only 10 patients underwent complete excision. Hakan T et al ${ }^{(9)}$ reported that in their series that $19 \%$ of their patients treated by means of aspiration underwent the procedure more than once. Mut et al ${ }^{\left({ }^{10}\right)}$ retrospectively analysed 20 patients who had large, solitary, capsulated, and superficially located lobar abscesses and compared the efficiency of two different surgical approaches i.e. aspiration vs capsular excision and their impact on length of hospital stay. The hospital stay was longer in the aspiration group 46.6 days ( $\mathrm{SD} \pm 24$ ) compared to the excision group 22.4 days (SD \pm 13 ) which was a significant difference $(p=0.01)$. Similarly, in our study, it was observed that length of hospital stay in excision group was less as compared to medical group and aspiration group.

\section{CONCLUSION}

The overall incidence of bacterial brain abscess has remained relatively constant despite improved treatment of underlying systemic infections and development of more effective antibiotics. Majority of patients are adults ( $>18$ years of age), the commonest age group being 20-29 years, with males outnumbering females. The most typically isolated microorganisms are Streptococcus and Staphylococcus species. CT (plain and contrast) is easily and widely used for confirming the diagnosis as well as for follow-up after the treatment period. MR imaging is utilized when diagnosis is in doubt. The treatment of brain abscesses involves both medical and surgical modalities. The nature of the abscess, its anatomic location, the number of abscesses and their size and stage, as well as the age and initial neurological status of the patient all influence the treatment strategy. Thirdgeneration cephalosporins and metronidazole are the most commonly used antimicrobial agents in the treatment of brain abscesses. Use. Prophylactic antiepileptic therapy is strongly recommended. The aspiration is the commonest procedure performed. Excision of abscess capsule was superior to aspiration in terms of efficiency of surgical intervention and postoperative cost of the treatment in a highly selected group of brain abscesses. A larger study with a greater number of patients is required for meaningful statics.

\section{REFERENCES}

1. Poffenbarger, G. J. "Management of solid intracranial abscess." Textbook of Neurological Surgery: Principles and Practice (2003): 3142-3150.

2. Ramamurthi, Ravi, and K. Sridhar. ",Trans-sulcal Approach to Intracerebral Lesions"." Textbook of Operative Neurosurgery 2 (2005): 335-337.

3. Rengachary, Setti S., and Richard G. Ellenbogen, eds. Principles of neurosurgery. Edinburgh:: Elsevier Mosby, 2005.

4. Yang SY. Brain abscess: A review of 400 cases. J Neurosurg. 1981;55:794-9.

5. Douglas and Bennett principles and practice of... Mandell, Raphael Dolin, and Gerald L. Mandell. Principles and practice of infectious diseases. Churchill Livingstone, 1995.

6. Çavuşoglu, Halit, Ramazan Alper Kaya, Osman Nuri Türkmenoglu, Ibrahim Çolak, and Yunus Aydin. "Brain abscess: analysis of results in a series of 51 patients with a combined surgical and medical approach during an 11 -year period." Journal of neurosurgery (2008):vol 6.

7. Beller A. J. A. Sahar, and I. Praiss. "Brain abscess: Review of 89 cases over a period of 30 years." Journal of Neurology, Neurosurgery \& Psychiatry 36, no. 5 (1973): 757-768

8. Schmidek, H. H. "Suppurative intracranial infections." Schmidek and Sweet's operative neurosurgical techniques indications, methods, and results, 5th edn. Elsevier, Philadelphia (2006): 1591-1599.

9. Hakan, Tayfun, Nurgül Ceran, İlknur Erdem, Mehmet Zafer Berkman, and Paşa Göktaş. "Bacterial brain abscesses: an evaluation of 96 cases." Journal of Infection 52, no. 5 (2006): 359-366

10. Mut M, Hazer B, Narin F, Akalan N, Ozgen T. Aspiration or capsule excision? Analysis of treatment results for brain abscesses at single institute. Turk Neurosurg. 2009;36-41. 